\title{
Choice, Consideration Sets, and Attribute Filters
}

\author{
By Mert KimyA*
}

\begin{abstract}
It is well known that decision makers do not always consider all of the available alternatives when making a choice. When the alternatives have attributes, these attributes provide a natural way to form the consideration set. I assume a procedure in which the decision maker uses the relative ranking of the alternatives on each attribute to reduce the size of the choice set. I provide a characterization of the procedure and illustrate how to identify the underlying preference and consideration set. The model explains certain choice anomalies such as the attraction and the compromise effects. (JEL C78, D11)
\end{abstract}

\begin{abstract}
uppose you are searching online for a hotel in a popular destination. Once you $\checkmark$ enter the dates and the city, you will realize that there are too many alternatives to choose from. Therefore, you will probably use the filters the website conveniently provides for you. A typical website has filters on price, star rating, and review rating. Most likely, you will use these filters to remove the hotels that rank poorly on some of these dimensions. Imagine removing hotels with a review rating less than 6 out of 10 and with a price more than $\$ 150$. Having removed some of the available alternatives this way, you would then select your preferred option from those that remain.

The idea that individuals first form a consideration set and then maximize the preference on that consideration set is not new. For an early example in the economics literature see Masatlioglu, Nakajima, and Ozbay (2012), who study preference maximization with attention filters. Although there have been many models that characterize how the consideration sets might be constructed, one thing that has been overlooked is that in many situations, as in the hotel choice example above, alternatives have observable attributes. When this is the case, it is natural to assume that the decision maker (DM) uses the observable attributes to form the consideration set.

I model a DM who has a stable preference over a set of alternatives. The alternatives have a set of attributes, and there is an objective ranking of alternatives on each attribute that all individuals agree on. This is in the spirit of the hotel choice example above, where the observable attributes such as price and review rating and the rankings over these attributes are all objective.
\end{abstract}

\footnotetext{
* Department of Economics, Koç University, Rumeli Feneri Yolu, Sarıyer 34450, Istanbul, Turkey (email: mkimya@ku.edu.tr). I am grateful to Mark Dean for guidance, support, and encouragement. I would also like to thank Geoffroy de Clippel, İnsan Tunalı, seminar participants at Koç University, seminar participants at Bilkent University, and two anonymous referees for helpful comments and suggestions.

Go to https://doi.org/10.1257/mic.20150235 to visit the article page for additional materials and author disclosure statement $(\mathrm{s})$ or to comment in the online discussion forum.
} 
Although individuals agree on the attribute rankings, they might disagree on the overall ranking of alternatives, i.e., the preferences might differ. Nevertheless, given the objectively defined attributes, it is sensible to assume that the preferences are monotonic in the sense that if an alternative $x$ dominates alternative $y$, that is, if $x$ ranks higher than $y$ in every attribute, then $x$ is preferred to $y$.

For a given set of alternatives with objectively defined attributes, the DM sets an unobservable threshold on each attribute and does not consider any alternative that stays below these thresholds. For instance, she does not consider hotels that are more expensive than $\$ 150$ or hotels with a review rating less than 6 out of 10 . Once she eliminates these alternatives, she maximizes her preference on the set of remaining alternatives.

Any theory with a situation dependent criterion derives its testable implications from the way this criterion cannot vary arbitrarily across situations. In my model, this criterion is how the thresholds are determined. I require that when we add an alternative $x$ above the threshold of attribute $i$, the threshold can only increase, but never to the point that it leads the DM to eliminate some alternative $y$ that ranks better than $x$ in attribute $i$. Similarly, when we add an alternative $x$ below the threshold of attribute $i$, the threshold can only decrease, but never to the point that it leads the DM to consider some alternative $y$ that ranks worse than $x$ in attribute $i$.

For instance, suppose the DM eliminates hotels that have a review rating less than 6. If we include additional hotels that have a review rating more than 6 , then the DM can only move the threshold up. But the DM cannot overreact by moving the threshold above alternatives that have a review rating higher than every alternative included in the set. Similarly, if we add hotels that have a review rating less than 6 , then the DM can only move the threshold down. But the DM cannot overreact by moving the threshold below alternatives that have a review rating lower than every alternative included in the set.

This restriction captures the intuitive notion that if a set improves in one attribute, then the DM becomes more discerning on this attribute. This intuitive appeal is evidenced by the variety of choice procedures that satisfy this restriction (see the examples in Section IB). Furthermore, this restriction also avoids certain implausible behaviors. An example is the choice anomaly in which the introduction of an asymmetrically dominated alternative leads to a choice reversal from a dominant alternative to a nondominant alternative. This pattern of behavior is the opposite of the attraction effect (see Huber, Payne, and Puto 1982), and it has no empirical support (see Simonson 1989; Huber, Payne, and Puto 1982; also see Section IIC).

I call the choice procedure that satisfies these conditions choice through attribute filters (CAF). The model has several interpretations:

- As in the hotel choice example, the set might contain too many elements to consider, in which case the DM might use the objectively defined attributes to reduce the size of the set.

- The DM might be using a "rule of thumb" to eliminate certain alternatives based on their attribute rankings even when the size of the set is small. For instance, the DM might have a fixed cutoff (see Example 4 in Section IB) or she might use satisficing on one of the attribute rankings (see Example 5 in 
Section IB) or she might eliminate nondominant alternatives in a set containing asymmetrically dominated alternatives (see Sections IIC and III). Further examples of this kind are available in Section IB.

I use the term "multi-criteria choice data" to refer to the dataset that includes the objective attributes and the rankings on these attributes. Data that includes observable attributes has been used before both in theoretical and experimental papers (see de Clippel and Eliaz 2012; Gabaix et al. 2006; Huber, Payne, and Puto 1982; Simonson 1989; and Tversky and Simonson 1993). Multicriteria choice data is easy to collect in many settings, especially in controlled laboratory environments, where the alternatives can be defined with a set of attributes. ${ }^{1}$

I characterize the conditions under which multi-criteria choice data is consistent with CAF. This is done by determining what revealed consideration and revealed preference mean in the context of my model. The choice of one alternative over another does not indicate preference, as the DM may not have considered the unchosen alternative. However, if we can establish that the unchosen alternative is considered (revealed consideration), this would imply a preference in favor of the chosen alternative (revealed preference).

For example, suppose the choice of the DM is consistent with CAF and she chooses $x$ from some set $S$. This would trivially imply that $x$ is considered in $S$. Now assume that as we go from set $S$ to set $T$, we are adding alternatives that rank worse than $x$ and removing alternatives that rank better than $x$ in each attribute. By the restriction on the thresholds, $x$ should be considered in set $T$ (revealed consideration). Furthermore, if $y$ is chosen from $T$, this would imply that $y$ is revealed to be preferred to $x$ (revealed preference). The necessary and sufficient condition for multi-criteria choice data to be consistent with CAF is the acyclicity of the revealed preference.

I start with the formal description of the model in Section I. In Section IIA, I provide the necessary and sufficient conditions for the data to be consistent with CAF. Section IIB fully characterizes what we can learn about the DM's preference and the consideration set if the choice is CAF. In Section IIC, I show that CAF model is able to explain certain consistently observed choice anomalies such as the attraction and the compromise effects. While explaining these effects, CAF is also inconsistent with the "opposites" of each of these effects.

In Section III, I take an axiomatic approach and directly put restrictions on the kind of choice anomalies allowed in the data. I restrict attention to choice functions that only allow for choice reversals in the spirit of the attraction and the compromise effects. That is, if inclusion of an alternative $z$ leads to a choice reversal from $x$ to $y$, then either $y$ asymmetrically dominates $z$ or $y$ is intermediate between $x$ and $z$. It turns out that this model is completely characterized by a simple class of CAF, in which there is at most a single undominated alternative in each consideration set. Section IV contains the literature review and also formally shows that the model is

\footnotetext{
${ }^{1}$ It is important to note that neither multi-criteria choice data nor CAF require that all of the available attributes and the rankings on these attributes are observable. The only requirement is that those attributes that are used for the construction of the consideration set are observable.
} 
independent of certain prominent alternatives. Appendix A provides additional auxiliary results and Appendix B contains the proofs.

\section{Model}

\section{A. Multicriteria Choice Data}

Let $Z \subset \mathbb{R}_{++}^{k}$ be a finite set of alternatives. Denote by $\mathcal{Z}$ the set of all non-empty subsets of $Z$. Each $l \in\{1,2, \ldots, k\}$ denotes an attribute and $z_{l}$ denotes the value of alternative $z$ on attribute $l$. $^{2}$

For convenience, I assume no ties in attribute rankings, i.e., for any $z, z^{\prime} \in Z$ with $z \neq z^{\prime}$ and $l \in\{1,2, \ldots, k\}$, we have $z_{l} \neq z_{l}^{\prime}$.

A choice function is a mapping $c: \mathcal{Z} \rightarrow Z$ such that $c(S) \in S$ for all $S \in \mathcal{Z}$. It is interpreted as the choice of the DM from each set. Multicriteria choice data includes $c$.

The assumption of no ties in attribute rankings and the restriction to choice functions is made for convenience. In Appendix A, I show that the main results are still valid with minor modifications even if we relax these assumptions by looking at choice correspondences and ties in attribute values.

\section{B. Choice through Attribute Filters}

The DM has a consideration set mapping $\Gamma: \mathcal{Z} \rightarrow \mathcal{Z}$, where $\Gamma(S) \subseteq S$ for each $S \in \mathcal{Z}$. Here, $\Gamma(S)$ denotes the set of alternatives she considers.

We say that $x$ dominates $y, x>_{D} y$, if $x_{i}>y_{i}$ for all $i \in\{1,2, \ldots, k\}$. The DM has a complete, transitive, strict, and monotene preference $\succ$ on $Z$, where a preference is monotone if $x>_{D} y$ implies $x \succ y \cdot{ }^{3}$ Once she forms the consideration set, she is assumed to maximize $\succ$ on this consideration set. Both the preferences and the consideration sets are unobservable.

Without any restriction on $\Gamma$, we can rationalize any dataset by simply assuming that only the chosen alternative is considered in each set, i.e., we can claim that $\Gamma(S)=c(S)$ for all $S$.

The existence of attributes and the rankings over these attributes provides a natural way to form the consideration set. For each set $S$, the DM sets a threshold $t^{S} \in \mathbb{R}^{k}$ and she only considers those alternatives that stay above the threshold. That is, for each $S \in \mathcal{Z}$, the consideration set is given by $\Gamma(S)=\left\{z \in S \mid z>t^{S}\right\}$. $\left.\right|^{4}$

Furthermore, the thresholds do not move arbitrarily. I require that when we add an alternative $x$ above the threshold of attribute $i$, the threshold on attribute $i$ can only increase, but never to the point that it leads the DM to eliminate some alternative $y$ with $y_{i}>x_{i}$. Similarly, when we add an alternative $x$ below the threshold of attribute $i$, the threshold can only decrease, but never to the point that it leads the DM to consider some alternative $y$ with $x_{i}>y_{i}$.

\footnotetext{
${ }^{2}$ The model is ordinal. Hence, only the induced order matters.

${ }^{3}$ Remark 2 in Appendix A discusses the implications of dropping the monotonicity condition.

${ }^{4}$ Note that $z>t$ if $z_{i}>t_{i}^{S}$ for $i=1, \ldots, k$.
} 
That is, if $T=S \cup x$ and if $x_{i} \geq t_{i}^{S}$ for some attribute $i$, then $t_{i}^{T} \geq t_{i}^{S}$ (if we add an alternative above the threshold, the threshold can only increase). Furthermore, $t_{i}^{T}$ cannot overreact by eliminating an alternative $y$ with $y_{i}>x_{i}$.

Similarly, if $T=S \cup x$ and if $x_{i} \leq t_{i}^{S}$ for some attribute $i$, then $t_{i}^{T} \leq t_{i}^{S}$ (If we add an alternative below the threshold, the threshold can only decrease). Furthermore, $t_{i}^{T}$ cannot overreact by saving an alternative $y$ with $x_{i}>y_{i}$.

The following definition formalizes these restrictions. For any $x \in Z$, let $\bar{x}_{i}(S)$ $=\min _{\left\{z \in S \mid z_{i}>x_{i}\right\}} z_{i}$ be the value of the first alternative in $S$ that ranks above $x$ in attribute $i$. If there does not exist such an alternative, let $\bar{x}_{i}(S)=\infty$. Similarly, let $\underline{x}_{i}(S)=\max _{\left\{z \in S \mid z_{i}<x_{i}\right\}} z_{i}$ be the value of the first alternative in $S$ that ranks below $x$ in attribute $i$. If there does not exist such an alternative, let $\underline{x}_{i}(S)=-\infty$.

DEFINITION 1: A consideration set mapping $\Gamma$ is an attribute filter if for each $S \in \mathcal{Z}$, there exists a threshold $t^{S} \in \mathbb{R}^{k}$ such that

(A) $\Gamma(S)=\left\{z \in S \mid z>t^{S}\right\}$.

(B) If $T=S \cup x$, where $x \in Z$ and $x_{i} \geq t_{i}^{S}$, then $\bar{x}_{i}(S)>t_{i}^{T} \geq t_{i}^{S}$.

(C) If $T=S \cup x$, where $x \in Z$ and $x_{i} \leq t_{i}^{S}$, then $\underline{x}_{i}(S) \leq t_{i}^{T} \leq t_{i}^{S}$.

Condition $(A)$ states that only those alternatives that are above the threshold are considered in each set. Condition $(B)$ requires that if an alternative is added above the threshold in attribute $i$, then the threshold can only move up, but any alternative ranking above the included alternative should still stay above the threshold. Similarly, condition $(C)$ requires that if an alternative is added below the threshold in attribute $i$, then the threshold can only move down, but any alternative ranking below the included alternative should still stay below the threshold. The implication of removing the latter two conditions will be discussed in Appendix A.

The following examples illustrate the wide range of procedures that lead to an attribute filter. I will show how to set these up as attribute filters in Appendix B.

Example 1 (Rational Choice): Consider everything.

Example 2 (Top $n$ ): Consider everything if the set contains less than $n$ elements. If the set contains more than $n$ elements, then consider the best $n$ elements according to a single attribute ranking. For example, consider the $n$ cheapest alternatives.

Example 3 (Eliminate Worst $n$ on Each Attribute): The DM eliminates the worst $n$ alternatives in each attribute ranking. If doing this leaves no options, then she eliminates the worst $n-1$ instead. If still everything is eliminated, then she eliminates the worst $n-2$ instead, and so on.

Example 4 (Fixed Cutoff): The DM has a fixed cutoff $a_{i}^{*}$ for each attribute $i$. She eliminates the alternatives that stay below the cutoff at each attribute ranking. The DM also has a fixed ordering of attributes and if everything is eliminated, then she removes the cutoff of the attributes in that order until the set contains at least one element. 
Example 5 (Satisficing (Simon 1955)): The DM has a stable preference and a threshold $a^{*} \in Z$. She chooses an attribute $i$, then she considers the best element in attribute $i$. If this element is not preferred to $a^{*}$, then she also considers the second best element in attribute $i$. She continues doing this until she finds an alternative that is preferred to $a^{*}$. If there is nothing preferred to $a^{*}$, then she considers everything. For example, she considers the cheapest alternative. If it is better than $a^{*}$, then she does not consider anything else. If not, then she considers the second cheapest alternative, and so on.

It is important to note that attribute filters are independent of other prominent consideration sets used in the literature, such as attention filters (Masatlioglu, Nakajima, and Ozbay 2012) and competition filters (Lleras et al. 2017). For instance, Example 3 above is neither an attention filter nor a competition filter, but it is an attribute filter. However, if the DM considers only the top alternative in each attribute ranking, then the resulting consideration set will not be an attribute filter, but it will be an attention filter and a competition filter (see Section IV for the definitions of attention and competition filters and a formal argument showing independence).

Now, we are ready to define CAF.

DEFINITION 2 (Choice Through Attribute Filters): A choice function $c$ is CAF if there exists a strict, complete, transitive, and monotone preference relation $\succ$ over $Z$ and an attribute filter $\Gamma$ such that $c(S)$ is the $\succ$-best element in $\Gamma(S)$ for every $S \in \mathcal{Z}$.

\section{Representation and Identification}

What are the necessary and sufficient conditions for $c$ to be CAF? And if $c$ is $\mathrm{CAF}$, then what can we confidently conclude about the preference and the consideration set of the DM? This section aims to answer these questions.

\section{A. Representation}

Suppose that $c$ is CAF and $c(S)=x$ for some set $S$. If $y \in S$, then we cannot immediately conclude that $x$ is preferred to $y$, as $y$ might not be considered. But, if we can conclude that $y$ is considered in $S$, then we are able to say that $x$ is preferred to $y$.

Now suppose that for some attribute $i$, there exists a set $T^{i}$ such that either $y=c\left(T^{i}\right)$ or $y_{i}>c\left(T^{i}\right)_{i}$. Furthermore, assume that set $S$ can be obtained from $T^{i}$ by removing alternatives that rank better than $c\left(T^{i}\right)$ and by adding alternatives that rank worse than $y$ in attribute $i$.

When we remove alternatives that rank better than $c\left(T^{i}\right)$, the threshold of attribute $i$ cannot increase to the point where it leads the DM to eliminate $c\left(T^{i}\right)$ in this new set. Since $y$ ranks better than $c\left(T^{i}\right)$ in attribute $i, y$ should also stay above the threshold of attribute $i$. Set $S$ is obtained from this new set by adding alternatives that rank worse than $y$ in attribute $i$. By the restriction on the thresholds, this implies that the threshold of attribute $i$ stays below $y$ in set $S$. Hence, $y$ is not eliminated by attribute $i$ in set $S$. 
If we can find such a set $T^{i}$ for each attribute $i$, then we can conclude that $y$ is not eliminated by any attribute and hence is considered in set $S$. Since $c(S)=x$, this would imply that $x$ is preferred to $y$.

Also, if $x>_{D} y$, we can trivially conclude that $x$ is preferred to $y$. Below, I will define the relation $P$ based on these observations.

DEFINITION 3: Let $x, y \in Z$ with $x \neq y$. We say $x P$ if $x>_{D} y$ or if there exists a set $S$ including $y$ such that $c(S)=x$ and for each attribute $i$ there exists a set $T^{i}$ such that either $y=c\left(T^{i}\right)$ or $y_{i}>c\left(T^{i}\right)_{i}$, and

- If $z \in T^{i} \backslash S$ then $z_{i}>c\left(T^{i}\right)_{i}$.

- If $z \in S \backslash T^{i}$ then $y_{i}>z_{i}$.

If $c$ is CAF, then the argument above the definition shows that $P$ should be acyclic. It turns out that acyclicity of $P$ is also sufficient for $c$ to be CAF.

THEOREM 1: A choice function $c$ is CAF if and only if $P$ is acyclic.

We have already seen that if $c$ is CAF then $P$ must be acyclic. For the other direction, assume that $P$ is acyclic. Since $P$ is also monotone, we can extend it to a complete and monotone preference relation. Then, we can construct the thresholds so that they eliminate every alternative that does not satisfy the condition in Definition 3. This way, in any set $S$, any alternative $y \in S$ such that $y P c(S)$ will not be considered and the choice will maximize the preference within the consideration set. Then, the proof boils down to showing that these constructed thresholds satisfy the condition of being an attribute filter. For details, see Appendix B.

\section{B. Identification}

In this section, I will fully characterize what we can learn about the DM's preference (revealed preference) and the consideration set (revealed consideration) if the choice is CAF. I will follow Masatlioglu, Nakajima, and Ozbay (2012) in defining the revealed preference and consideration.

DEFINITION 4: Suppose a choice function c is CAF. Let the set of consideration set and preference pairs $\left\{\left(\Gamma^{1}, \succ^{1}\right),\left(\Gamma^{2}, \succ^{2}\right), \ldots,\left(\Gamma^{m}, \succ^{m}\right)\right\}$ be the exhaustive list of representations consistent with CAF. Then

- $x$ is revealed to be preferred to $y$ if and only if $x \succ^{j} y$ for all $j=1,2, \ldots, m$.

- $x$ is revealed to be considered in $S$ if and only if $x \in \Gamma^{j}(S)$ for all $j=1, \ldots, m$.

This is a conservative definition, as it requires all representations to agree to conclude something about the preference or the consideration set of the DM.

The discussion above has shown that if $x P y$, then $x$ is revealed to be preferred to $y$. Since the underlying preference is transitive, we can also conclude that if $x P y$ and y $P z$, then $x$ is revealed to be preferred to $z$. This means that if we denote by $P_{R}$ the 


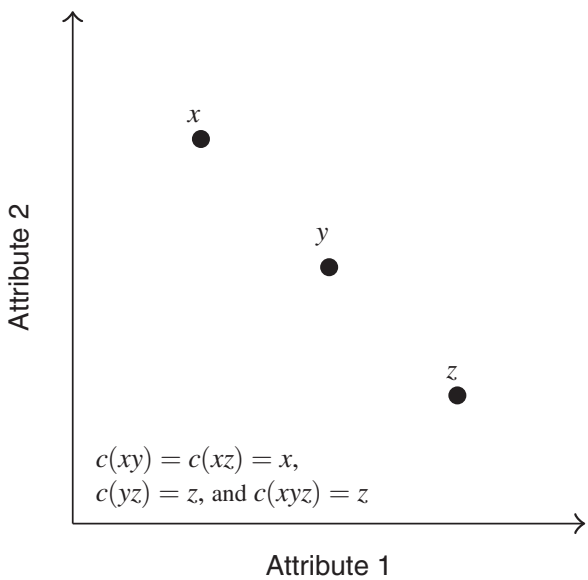

Figure 1. Data Corresponding to Example 6

Table 1-Revealed Preference and Consideration for Example 6

\begin{tabular}{|c|c|c|c|c|}
\hline $\bar{S}$ & $\{x, y\}$ & $\{x, z\}$ & $\{y, z\}$ & $\{x, y, z\}$ \\
\hline Revealed preference & \multicolumn{4}{|c|}{$x \succ z$} \\
\hline Revealed consideration & $\{x\}$ & $\{x, z\}$ & $\{z\}$ & $\{z\}$ \\
\hline Actual $\Gamma(S)$ & $\{x, y\}$ & $\{x, z\}$ & $\{y, z\}$ & $\{y, z\}$ \\
\hline
\end{tabular}

transitive closure of $P$, then $x$ is revealed to be preferred to $y$ if $x P_{R} y$. It turns out that $P_{R}$ is the revealed preference in this model.

Furthermore, we have seen that for $x \in S$, if we can find a set $T^{i}$ for each attribute $i$ that satisfies the condition in Definition 3, then $x$ is revealed to be considered in $S$. It turns out that this is the revealed consideration in this model.

THEOREM 2: Suppose a choice function $c$ is $C A F$, then

- $x$ is revealed to be preferred to $y$ if and only if $x P_{R} y$.

- $x$ is revealed to be considered in set $S$ if and only if for each attribute i there exists a set $T^{i}$ such that $x=c\left(T^{i}\right)$ or $x_{i}>c\left(T^{i}\right)_{i}$, and

- If $z \in T^{i} \backslash S$, then $z_{i}>c\left(T^{i}\right)_{i}$.

-If $z \in S \backslash T^{i}$, then $x_{i}>z_{i}$.

The theorem above shows what we can confidently say about the consideration set and the preference of the DM if the choice is CAF. The example below demonstrates revealed consideration and preference.

Example 6: Suppose that $Z=\{x, y, z\}$, there are two attributes and the attribute rankings are given in Figure 1. Suppose the DM has the following (unobservable) preference, $x \succ z \succ y$, and uses the following choice procedure. She always considers two alternatives and she does this by eliminating the worst $|S|-2$ alternatives 
in attribute 1, if $|S| \geq 3$. Then, she maximizes her preference in the corresponding consideration set. The data corresponding to this example is in Figure 1.

It is easy to see that this choice is consistent with CAF. Table 1 lists the revealed preference and consideration corresponding to this example.

Consider the set $\{x, z\}$. As the choice is $x$, we know that $x$ is considered in this set. Note that $z$ stays above the threshold of attribute 2 in $\{x, y, z\}$, because it is chosen. As we go from $\{x, y, z\}$ to $\{x, z\}$, we are removing an alternative above $z$ in attribute 2 , so $z$ should again stay above the threshold of attribute 2 in $\{x, z\}$. Furthermore, as $z$ ranks higher than $x$ in attribute 1, we also know that $z$ stays above the threshold of attribute 1 . Hence, $z$ is considered in $\{x, z\} .5$ By Definition 3, we have $x P z$ and by Theorem 2, $x$ is revealed to be preferred to $z$. It turns out that this is the revealed preference we can get in this example. A similar exercise can be repeated to obtain every cell of Table 1 .

Note that for each set, the set of alternatives that are revealed to be considered is a subset of the actual consideration set. This will always be the case because revealed consideration includes only those alternatives that are included in the consideration set of every possible representation. Furthermore, in this example the revealed preference is incomplete, which implies that there are multiple preferences that can rationalize the choice. For example, the actual consideration set and the preference of the DM would rationalize the choice, but also the following would: $x \succ y \succ z$, $t^{S}=(0,0)$ for $S \in\{\{x, z\},\{x, y\}\}$, and $t^{S}=\left(y_{1}, 0\right)$ for $S \in\{\{x, y, z\},\{y, z\}\}$, leading to $\Gamma(x y z)=\Gamma(y z)=\{z\}, \Gamma(x y)=\{x, y\}$, and $\Gamma(x z)=\{x, z\}$.

An example that is not consistent with CAF is given in the next subsection.

\section{Choice Anomalies and CAF}

The attraction and the compromise effects are two of the most consistently observed violations of the Weak Axiom of Revealed Preference (WARP). The attraction effect was first demonstrated by Huber, Payne, and Puto (1982). It refers to the observation that the inclusion of an asymmetrically dominated alternative may lead to a shift in choice in favor of a dominant alternative. The compromise effect was first demonstrated by Simonson (1989). It refers to the observation that the inclusion of an extreme alternative may lead to a shift in choice in favor of an intermediate alternative. A typical dataset that corresponds to these effects can be found in Figure 2.

CAF is able to explain both of these as resulting from the same choice procedure. First, consider the data corresponding to the attraction effect. This data can be rationalized with the following preference, $x \succ y \succ z$ and the following thresholds, $t^{S}=\left(z_{1}, z_{2}\right)$ if $S \in\{\{y, z\},\{x, y, z\}\}$, and $t^{S}=(0,0)$ otherwise. The elimination sets satisfy the restrictions and $c$ maximizes the preference in the corresponding consideration set. The compromise effect can be rationalized with $x \succ z^{\prime} \succ y$ and with the following thresholds, $t^{S}=(0,0)$ if $|S|<3$ and $t^{S}=\left(x_{1}, z_{2}^{\prime}\right)$ if $S=\left\{x, y, z^{\prime}\right\}$.

The idea is simple. In the binary choice between $x$ and $y$ everything is considered, so the DM maximizes her preference in this set. In the attraction effect, when we

\footnotetext{
${ }^{5}$ Rewriting in terms of the notation in Theorem 2, just take $T^{1}=\{x, z\}$ and $T^{2}=\{x, y, z\}$.
} 

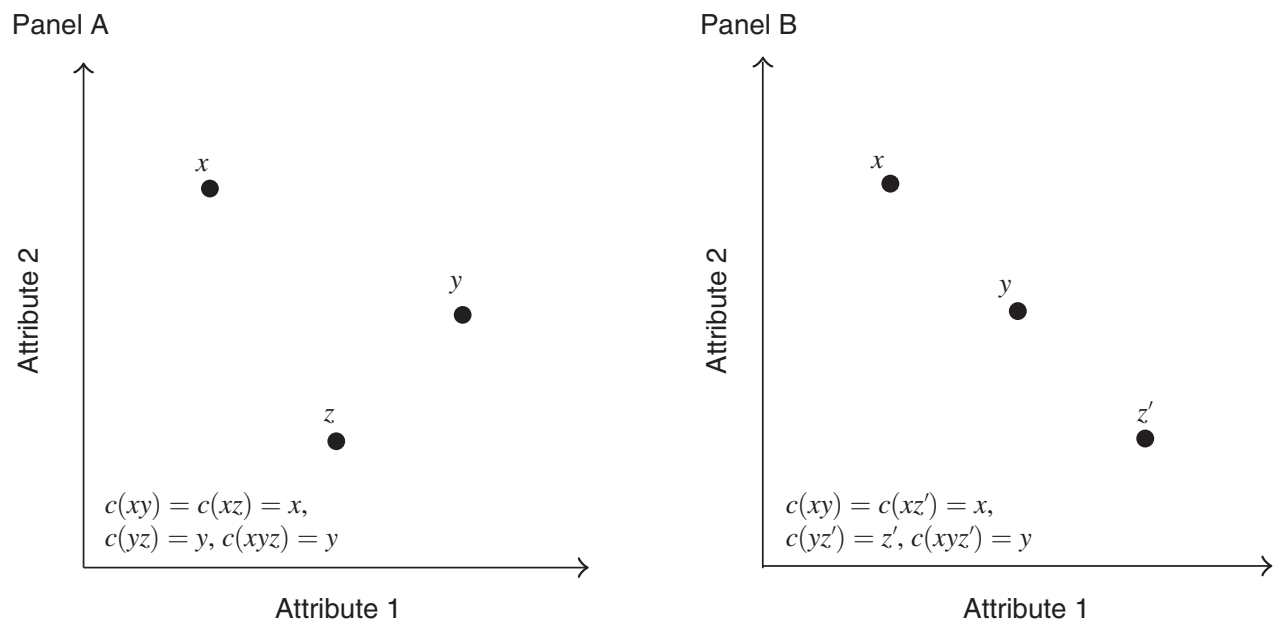

Figure 2. Data Corresponding to the Attraction Effect (on the left) AND THE COMPROMISE EFFECT (on the right)

include $z$, it makes $x$ look relatively poor on attribute 1 , which leads $x$ to be eliminated and $y$ to be chosen. In the compromise effect, when faced with all three alternatives, she eliminates the extremes, which leads to the choice of the intermediate one.

It is not helpful for a model to explain such anomalies if it does not put reasonable restrictions on what is consistent with the model. We will now see that neither the opposite of attraction nor the opposite of compromise can be explained with CAF. So, assume that $c(x y)=y$ and $c(x y z)=c\left(x y z^{\prime}\right)=x$ for the alternatives given in Figure 2 .

The following argument shows that the opposite of attraction is not compatible with CAF. First note that, since $c(x y)=y, y$ is considered in $\{x, y\}$. As we go from $\{x, y\}$ to $\{x, y, z\}$ we are adding an alternative worse than $y$ in attribute 2 , hence $y$ stays above the threshold of attribute 2 in $\{x, y, z\}$. Also, $x$ is chosen in $\{x, y, z\}$ and $x$ ranks lower than $y$ in attribute 1 , so $y$ also stays above the threshold of attribute 1 . But then, $y$ is revealed to be considered in $\{x, y, z\}$, implying $x P y$.

As $c(x y z)=x, x$ is considered in $\{x, y, z\}$. As we go from $\{x, y, z\}$ to $\{x, y\}$ we are removing an alternative that ranks better than $x$ in attribute 1 , so $x$ stays above the threshold of attribute 1 in $\{x, y\}$. Also, $y$ is chosen in $\{x, y\}$ and $y$ ranks lower than $x$ in attribute 2, so $x$ also stays above the threshold of attribute 2. Then, $x$ is revealed to be considered in $\{x, y\}$, implying $y P x$. But then, we have $x P$ y $P x$, hence the data is inconsistent with CAF. The same argument, with $z$ replaced with $z^{\prime}$ also shows that the opposite of compromise is not compatible with CAF.

\section{Attraction or Compromise ${ }^{6}$}

The previous section has shown that CAF provides an explanation for the attraction and the compromise effects. Given this feature of the model, one might be

\footnotetext{
${ }^{6} \mathrm{I}$ am grateful to an anonymous referee for suggesting this section.
} 
interested in how these effects generalize to more than three alternatives and whether a class of attribute filters can be characterized with such generalizations.

In this section, I take an axiomatic approach and come up with a model that only allows for choice anomalies in the spirit of the attraction and the compromise effects. I show that this model is completely characterized by a particularly simple class of CAF.

Throughout this section I assume that there are only two attributes, i.e., $Z \subset \mathbb{R}_{++}^{2}$. This is because the attraction and the compromise effects are defined with two attributes, and there is no obvious way to define the compromise effect with more than two attributes.

ATTRACTION OR COMPROMISE: A choice function $c$ satisfies Attraction or Compromise if whenever $c(S)=x$ and $c(S \cup z)=y$, where $y \neq x$ and $y \neq z$, we have either

(i) $y>_{D} z$ and $x \ngtr_{D} z$ or

(ii) $x_{i}>y_{i}>z_{i}$ and $z_{j}>y_{j}>x_{j}$, where $i \neq j, i, j \in\{1,2\}$.

If choice satisfies this axiom, then any choice reversal will either be in favor of a dominant alternative when an asymmetrically dominated alternative is added to the set (i) or in favor of an intermediate alternative (ii). Hence, every choice anomaly is either in the spirit of the attraction effect or the compromise effect.

ATTRACTION CONSISTENCY: A choice function $c$ satisfies Attraction Consistency if whenever $c(S)=x, c(S \cup z)=y$, where $y>_{D} z, x \ngtr_{D} z$, and if there exists $z^{\prime} \in S$, where $z^{\prime} \neq y$ and $z^{\prime}>_{D} z$, then either

- $y>_{D} z^{\prime}$ or

- $x_{i}>y_{i}>z_{i}^{\prime}$ and $z_{j}^{\prime}>y_{j}>x_{j}$, where $i \neq j, i, j \in\{1,2\}$.

This axiom is a consistency condition on the "attraction effect." It requires that if $z$ leads to the "attraction effect" in favor of $y$, but if there is another alternative $z$ ' that dominates $z$, then it should be the case that either $y$ dominates $z^{\prime}$ or $y$ benefits from being in between $z^{\prime}$ and the previously chosen alternative $x$. In other words, $y$ either benefits from being dominant or from being intermediate.

MONOTONICITY: A choice function c satisfies Monotonicity if $c(S)=x$ implies that there does not exist $y \in S$ with $y>_{D} x$.

This axiom simply states that dominated alternatives cannot be chosen. It is not a completely innocuous axiom. Depending on what the attributes are it might be violated by certain plausible choice procedures. Nevertheless, if the attribute rankings are defined in accordance with the preference, then the axiom is indeed plausible. For instance, if price is an attribute, then the attribute values on this dimension should be defined as $x_{i}>y_{i}$ only if $x$ is cheaper than $y$. 
It turns out that these axioms completely characterize a particularly simple class of CAF. For any set $S \in \mathcal{Z}$, let $S^{\text {nd }} \subseteq S$ denote the set of undominated alternatives in $S$, i.e., $S^{\text {nd }}=\left\{z \in S \mid \nexists z^{\prime} \in S\right.$ with $\left.z^{\prime}>_{D} z\right\}$. CAF with total elimination (CAFTE) is CAF where $\Gamma(S)^{n d}$ includes a single alternative for all $S \in \mathcal{Z}$.

DEFINITION 5: A choice function $c$ is CAFTE if $c$ is CAF and $\left|\Gamma(S)^{\text {nd }}\right|=1$ for all $S \in \mathcal{Z}$.

CAFTE is CAF where each consideration set includes exactly one undominated alternative. It turns out that CAFTE is completely characterized by Attraction or Compromise, Attraction Consistency, and Monotonicity.

THEOREM 3: A choice function $c$ is CAFTE if and only if c satisfies Attraction or Compromise, Attraction Consistency, and Monotonicity.

It is straightforward to show that if $c$ is CAFTE, then $c$ satisfies the axioms. Basically, a simple case by case analysis proves that when only the chosen alternative is considered (excluding dominated alternatives), the movement of the thresholds consistent with CAF would only allow for choice reversals consistent with the three axioms above.

For the other direction, assume that $c$ satisfies the axioms. In any binary set, we can put both of the thresholds right below the chosen alternative (except when one of the alternatives is dominated). Since the axioms always change the choice toward the direction of movement allowed in Definition 1, as we add an alternative to a binary set, we can still move the thresholds right under the chosen alternative. With the same argument, we can keep moving the thresholds right under the chosen alternative in any set, except when there are dominated alternatives, in which case the dominated alternative limits the movement of the threshold. Hence, every undominated alternative can be eliminated in any set and the choice can be rationalized as CAFTE. See the formal proof in Appendix B for details.

An interesting example of a choice procedure that gives rise to data that is CAFTE is the fallback bargaining solution (see de Clippel and Eliaz 2012, Sprumont 1993, Brams and Kilgour 2001 and Kibris and Sertel 2007) with a deterministic tiebreaking rule.

Example 7 (Fallback Bargaining Solution): Let $s_{i}(x, S)=\left|\left\{y \in S \mid x_{i}>y_{i}\right\}\right|$ be the number of alternatives that rank worse than $x$ in attribute $i$ in set $S$. The fallback bargaining solution assigns to each set $S$ the set of alternatives that maximize the minimum of the two scores, i.e., $C^{f}(S)=\arg \max _{x \in S} \min _{i=1,2} s_{i}(x, S)$. The fallback bargaining solution is not a choice function as it might assign two alternatives to a set. Let $c^{i-d f}(S) \subseteq C^{f}(S)$ be the resulting choice function when ties are broken in favor of attribute $i . \overline{7}$

\footnotetext{
${ }^{7}$ Any deterministic tie-breaking rule would be CAFTE as long as there is no choice reversal when we include an alternative that is dominated by both alternatives in $C^{f}(S)$.
} 


\section{LEMMA 1: For any $i \in\{1,2\}$, the choice function $c^{i-d f}(S)$ is CAFTE.}

The result is not immediate. However, de Clippel and Eliaz (2012) have already shown that the fallback bargaining solution is closely related to the attraction and the compromise effects. In Appendix B, I prove this lemma by proving that the axioms in de Clippel and Eliaz (2012) that characterize the fallback bargaining solution imply the three axioms above..$^{8}$

See Figure 1 for an example of data that is CAF, but not CAFTE. We have already seen that this is CAF. However, it cannot be rationalized as CAFTE because the revealed consideration set for the set $\{x, z\}$ is $\{x, z\}$ itself and contains multiple undominated alternatives. Also, here we see that there is a choice reversal inconsistent with both the attraction and the compromise effects.

\section{Related Literature}

There is evidence that individuals do not consider all of the available alternatives when making a choice. This phenomena has been extensively studied in the marketing literature, see Hauser and Wernerfelt (1990), Roberts and Lattin (1991), and Wright and Barbour (1977) for examples.

There are several models where choice is made as a result of a two-stage procedure, the first stage of which can be interpreted as the formation of the consideration set. Manzini and Mariotti's (2007) rational shortlist method considers sequential maximization of several rationales. In Cherepanov, Feddersen and Sandroni's (2013) rationalization, the DM chooses the best alternative among the ones she can rationalize. In Manzini and Mariotti (2012), the DM categorizes the alternatives and she maximizes her preference among the ones in the "winning categories." These models are closely connected to each other, as their characterization involves similar axioms. In particular, a condition called weak WARP is necessary for the choice to be consistent with any of these models.

Masatlioglu, Nakajima, and Ozbay (2012) and Lleras et al. (2017) are more closely connected to this work. Just like this paper, they assume that the DM forms a consideration set and maximizes her stable preference on this consideration set. The models used in these studies differ from my model based on the properties of the consideration sets involved. Masatlioglu, Nakajima, and Ozbay (2012) studies attention filters, which require that if an alternative is not considered in a set, then the removal of this alternative does not affect the consideration set. Whereas Lleras et al. (2017) studies competition filters, which require that if an alternative is considered in a set, then it should be considered in every subset of this set including the alternative.

Caplin and Dean (2011); Caplin, Dean, and Martin (2011); and Masatlioglu and Nakajima (2013) look at the cases in which the consideration set is formed as a result of search through the set of alternatives. Both of these models look at data that is richer than choice data, in particular Caplin and Dean (2011) and Caplin, Dean, and Martin (2011) consider "choice process data," which includes how the choice

\footnotetext{
${ }^{8}$ Note that de Clippel and Eliaz (2012) characterizes the fallback bargaining solution, not the one with a deterministic tiebreaking rule. This is taken into account in the proof.
} 
changes through the process of search. The data Masatlioglu and Nakajima (2013) considers include the starting point of search. Both the motivation and the empirical implications of these models are quite different from CAF.

de Clippel and Eliaz (2012) models choice as the cooperative solution to a bargaining problem among the different selves of the individual. In their model, each attribute ranking is interpreted as the preference of one self. Each self assigns each alternative a score equal to the number of alternatives in the lower contour set of that alternative and the solution selects the alternative whose minimum score is highest. Hence, they do not model choice as a two-step procedure, and there is no underlying single preference ordering that represents the preference of the DM. As with CAF, their model can also explain both the attraction and the compromise effects.

Another model that is able to explain both the attraction and the compromise effects is Natenzon (forthcoming). The author takes a fundamentally different approach by using stochastic choice data, and he shows that these effects can arise from Bayesian updating.

Ok, Ortoleva, and Riella (2015) are able to explain the attraction effect, but not the compromise effect. According to their explanation, in the attraction effect, the dominated alternative acts as a reference for the agent and makes the dominant alternative more attractive. One important distinction between their model and mine is that they use standard choice data, whereas I use data that includes observable attributes. The approaches should be seen as complementary, since the former has the advantage of identifying the attraction effect and the attributes when they are subjective, while the latter can make firmer predictions when the attributes are objective.

Lombardi (2009) has a model that explains the attraction effect. In this model, the DM first eliminates the dominated alternatives according to a fixed, but possibly incomplete relation. From the remaining alternatives, she eliminates the ones whose lower contour set is strictly contained within another option's lower contour set.

Gerasimou (2016) also has a model that incorporates the attraction effect. In this model, the DM eliminates the dominated alternatives according to a partial order, but from the remaining set she chooses the ones that are preferred to some other alternative.

Mandler, Manzini, and Mariotti (2012) studies a choice procedure in which the DM sequentially goes through a checklist of properties, where at each stage she eliminates the alternatives that do not have the property. They show that this procedure has close connections to utility maximization. Unlike in this paper, the properties are unobservable and there is no underlying preference that the DM maximizes.

Tversky (1972) considers a probabilistic choice model in which the alternatives are characterized by a set of aspects. At each stage, an aspect is selected and all the alternatives that do not have this aspect are eliminated. This continues until all but one of the alternatives are eliminated. Unlike this model, this is a probabilistic model, and the DM does not have a stable preference. Furthermore, unlike Tversky (1972), the data I consider here allows for the movement of the threshold relative to the set under consideration.

Manzini and Mariotti (2014) considers a stochastic model of consideration set formation in which the DM considers each alternative with a certain unobservable probability and then maximizes her preference among the alternatives she considers. In their model, the elimination is done stochastically given set-independent probabilities, whereas in CAF elimination is deterministic and depends on the choice set. 
There are also applied theory papers that study the effects of limited consideration on markets. Eliaz and Spiegler (2011a) provides one of the earliest applications, where the firms can use costly marketing devices to influence the set of alternatives considered by the consumer. In a similar vein, Eliaz and Spiegler (2011b) studies a model in which products are used to influence what the consumer pays attention to.

In the rest of this section, I will provide a formal comparison to models that are most closely related to my paper. We say that a consideration set is an attention filter if when an alternative is not considered, then the removal of this alternative does not affect the consideration set (Masatlioglu, Nakajima, and Ozbay 2012). Formally, $\Gamma$ is an attention filter if for any $S, \Gamma(S)=\Gamma(S \backslash x)$ whenever $x \notin \Gamma(S)$. The corresponding choice procedure is called choice with limited attention (CLA).

We say that a consideration set is a competition filter if whenever an alternative is considered in a set $S$, then it will be considered in every subset of $S$ that includes this alternative (Lleras et al. 2017). Formally, $\Gamma$ is a competition filter if whenever $x \in S \subseteq T$ and $x \in \Gamma(T)$, then $x \in \Gamma(S)$. The corresponding choice procedure is called choice with limited consideration (CLC).

Remark 1: A consideration set might be an attribute filter, but fail to be an attention or a competition filter. Similarly, a consideration set might be an attention and/ or a competition filter, but fail to be an attribute filter.

Consider Example 3 in Section IB. Suppose that the DM eliminates the $n$ most expensive alternatives. This is not an attention filter, because when the $n$th most expensive alternative is removed from the set, an alternative that was originally considered will be eliminated. The same argument also shows that it is not a competition filter either, as an alternative is considered in a set, but not considered in one of its subsets.

Example 8 below is an example of an attention and a competition filter that is not an attribute filter.

Example 8 (Top $n$ on Each Attribute): The DM considers the best $n$ alternatives on each attribute ranking. It is clear that this is both an attention filter and a competition filter. To see that it is not an attribute filter, consider the following example. Let $Z=\{a, b, c\}$, there are two attributes and the rankings are $a_{1}>b_{1}>c_{1}$ and $c_{2}>b_{2}>a_{2}$. Suppose the DM considers the top alternative on each ranking, then $\Gamma(a b c)=\{a, c\}$. If this is an attribute filter, then $b$ is eliminated either by attribute 1 or 2 . But if $b$ is eliminated by attribute 1 , then by condition $(A), c$ is also eliminated. Similarly, if $b$ is eliminated by attribute 2 , then by condition $(A), a$ is also eliminated. So, this cannot be an attribute filter. And this proves Remark 1.

We have seen that attribute filters are independent of both attention and competition filters, but the question of whether the models are independent still remains.

Here, I will show that they are independent. The examples also show independence of CAF from Rationalization (Cherepanov, Feddersen, and Sandroni 2013) and Categorize then Choose (Manzini and Mariotti 2012). 9

\footnotetext{
${ }^{9}$ Note that the data I consider is richer than choice data, which all the other models mentioned here consider. In particular, I use multi-criteria choice data, which assumes that the attributes are observable. Whereas, all of the models discussed here use the regular choice data, which only includes the choice function.
} 


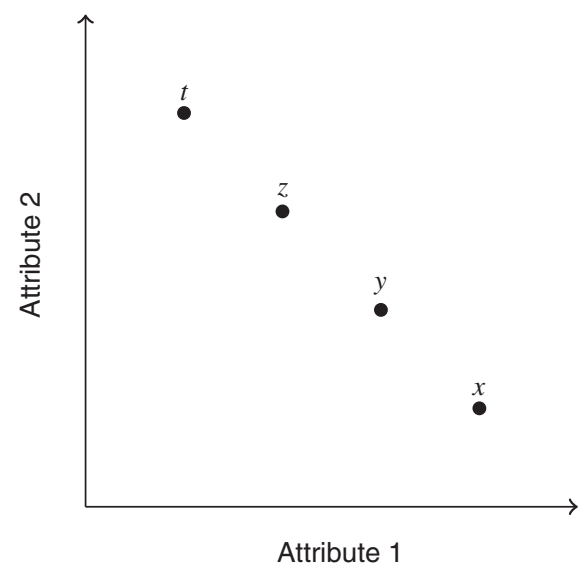

Figure 3. Attribute Rankings FOR ExAmPle 9

Example 9: Suppose that $Z=\{x, y, z, t\}$, there are two attributes, and the attribute rankings are given in Figure 3. Suppose the DM has the following preference, $x \succ y \succ t \succ z$ and uses the following choice procedure. In a binary set, she maximizes her preference. When given a set containing three or more alternatives, she eliminates the worst in each attribute ranking before maximizing her preference in the remaining set. This choice is consistent with CAF. But it is neither a CLA nor a CLC.

To see that it is not a CLA, observe that $c(x y z)=y$ and $c(y x)=x$. This implies that if the choice is a CLA, then the DM paid attention to $z$ in $\{x, y, z\}$ implying $y \succ z$. Similarly, $c(y z t)=z$ and $c(z t)=t$ implies $z \succ y$, a contradiction.

To see that this is not a CLC, observe that $c(x y z t)=y$ and $c(y z t)=z$. But if the choice is a CLC, then since $y$ is considered in $\{x, y, z, t\}, y$ is also considered in $\{y, z, t\}$, so $z \succ y$. Similarly, $c(y z t)=z$ and $c(y z)=y$ implies that $y \succ z$, a contradiction.

This example shows that CAF is not a special case of CLA and CLC. Another widely used axiom, which is a necessary condition in several models discussed in the literature review, is Weak WARP.

DEFINITION 6 (Weak WARP): A choice function c satisfies Weak WARP if $x \neq y$, $\{x, y\} \subseteq S \subseteq T$, and $c(x y)=c(T)=x$, then $c(S) \neq y$.

For $c$ to be consistent with the Rational Shortlist Method (Manzini and Mariotti 2007), Rationalization (Cherepanov, Feddersen, and Sandroni 2013) and Categorize then Choose (Manzini and Mariotti 2012), it has to satisfy Weak WARP. The above example also shows that $c$ might violate weak WARP, but still be consistent with CAF. To see this, observe that in the above example we have $c(x y z t)=y$, $c(z y)=y$, but $c(y z t)=z$. So, CAF is not a special case of any of these models.

Finally, Weak WARP is always satisfied when there are three alternatives. CLA and CLC also do not have any empirical content when there are three alternatives. But then, all of these models are consistent with the "opposites" of the attraction and the compromise effects studied in Section IIC, while CAF is not consistent 
with them. Hence, data might be consistent with CLA, CLC, Rationalization, and Categorize then Choose, but fail to be consistent with CAF. Furthermore, Example 9 has shown that data might be consistent with CAF, but fail to be consistent with CLA, CLC, Rationalization, and Categorize then Choose.

\section{Appendix A. Additional Results}

\section{A. Unobservable Attributes}

The model has no empirical content if the attributes are unobservable. Let $Z$ be any finite set of alternatives. ${ }^{10}$

DEFINITION 7: A choice function $c$ is a choice through attribute filters with unobservable attributes if there exists an integer $k>0$, a function $f: Z \rightarrow \mathbb{R}_{++}^{k}$ such that $(c, f(Z))$ is $C A F$.

PROPOSITION 1: Any choice function $c$ is a choice through attribute filters with unobservable attributes.

\section{PROOF:}

Take any $c$. I will show the result by constructing $|S|-1$ attributes for each set $S$. For any set $S$ and $x \in\{S \backslash c(S)\}$ construct an attribute ranking with the property that the elements in $S$ rank strictly higher than the elements in $Z \backslash S$ and $x$ is the alternative that ranks the lowest among the alternatives in $S$. In set $S$, the threshold on this attribute will be at $x$ and in any other set, the threshold will be $\epsilon$ below the lowest ranking alternative on this attribute, where $\epsilon>0$ is a small enough number such that $\left|z_{l}-z_{l}^{\prime}\right|>\epsilon$ for all $z \neq z^{\prime}$, for the constructed attribute $l$. So, $x$ and only $x$ will be eliminated using this attribute ranking in set $S$. And nothing will be eliminated by this attribute in any set $T \neq S$. We can do this for every alternative $x \in\{S \backslash c(S)\}$ and for every set $S \in \mathcal{Z}$.

It is easy to see that this leads to an attribute filter and $\Gamma(S)=c(S)$ for all $S \subseteq Z$. Hence, any linear order $\succ$ will rationalize the choice.

\section{B. Pre-attribute Filters}

A model with condition $(A)$ alone has little empirical content as any $c$ that satisfies Monotonicity will be rationalizable with such a model.

DEFINITION 8 (Pre-attribute Filter): A consideration set mapping $\Gamma$ is a pre-attribute filter iffor each $S \in \mathcal{Z}$, there exists a threshold $t^{S} \in \mathbb{R}^{k}$ such that

$$
\text { (A) } \Gamma(S)=\left\{z \in S \mid z>t^{S}\right\}
$$

\footnotetext{
${ }^{10}$ In the paper, we have taken $Z \subset \mathbb{R}^{k}$. This specification incorporates the attributes and the rankings on these attributes. Here, $Z$ is any finite set of alternatives.
} 
DEFINITION 9 (Choice Through Pre-attribute Filters): A choice function $c$ is a Choice Through Pre-attribute Filters if there exists a strict, complete, transitive, and monotone preference relation $\succ$ over $Z$ and a pre-attribute filter $\Gamma$ such that $c(S)$ is the $\succ$-best element in $\Gamma(S)$ for every $S \in \mathcal{Z}$.

PROPOSITION 2: Any choice function $c$ that satisfies Monotonicity is a Choice Through Pre-attribute Filters.

\section{PROOF:}

Take any $c$ that satisfies Monotonicity. Let $\succ$ be any complete, strict, monotone, and transitive preference relation. For every set $S$, let $t^{S}=\left(c(S)_{1}-\epsilon, c(S)_{2}-\right.$ $\left.\epsilon, \ldots, c(S)_{k}-\epsilon\right)$, where $\epsilon>0$ is a small enough number such that $\left|z_{l}-z_{l}^{\prime}\right|>\epsilon$ for all $z \neq z^{\prime}$ and $l \in\{1,2, \ldots, k\}$. The resulting consideration set is a pre-attribute filter; furthermore, everything in $S$ that does not dominate $c(S)$ is eliminated. Since $c$ is monotone, $c(S)$ maximizes $\succ$ in $\Gamma(S)$.

Remark 2: This brings the question of how monotonicity affects the main results in the paper. If we do not assume that the underlying preference is monotonic and if we drop the condition that $x P y$ if $x>_{D} y$ in Definition 3 , then all of the main results (Theorems 1 and 2) will still go through with similar proofs.

Remark 3: If we do not require the monotonicity of the underlying preference, then for this particular result, we will have that any $c$ will be a pre-attribute filter. The elimination sets in the proof will stay the same, but the preference will have the peculiar property that $x \succ y$ whenever $y>_{D} x$.

\section{Allowing for Indifference}

In this section, I will allow for indifference in the preference of the DM and in the attribute rankings. We will see that the main results go through with slight modification. As these are changes in the fundamentals, I will quickly go through the data and the choice procedure.

Here, $Z \subset \mathbb{R}_{++}^{k}$ is a finite set of alternatives, $\mathcal{Z}$ denotes the set of all non-empty subsets of $Z$. Now, it can be the case that $z_{i}=x_{i}$ for some $z, x \in Z$ with $z \neq x$ and some attribute $i$.

A choice correspondence is a mapping $C: \mathcal{Z} \rightarrow \mathcal{Z}$ such that $C(S) \subseteq S$ for all $S \in \mathcal{Z}$. It is interpreted as the choice of the DM from each set.

DEFINITION 10: A consideration set mapping $\Gamma$ is an attribute filter if for each $S \in \mathcal{Z}$, there exists a threshold $t^{S} \in \mathbb{R}^{k}$ such that

(A) $\Gamma(S)=\left\{z \in S \mid z>t^{S}\right\}$.

(B) If $T=S \cup x$ where $x \in Z$ and $x_{i} \geq t_{i}^{S}$, then $\bar{x}_{i}(S)>t_{i}^{T} \geq t_{i}^{S}$.

(C) If $T=S \cup x$ where $x \in Z$ and $x_{i} \leq t_{i}^{S}$, then $\underline{x}_{i}(S) \leq t_{i}^{T} \leq t_{i}^{S}$. 
We say that $x>_{D} y$ if $x_{i} \geq y_{i}$ for all $i \in\{1,2, \ldots, k\}$ and $x_{j}>y_{j}$ for some $j \in\{1,2, \ldots, k\}$. A preference relation $\succeq$ is monotone if $x>_{D} y$ implies $x \succ y$.

DEFINITION 11 (Choice Through Attribute Filters): A choice correspondence C is $C A F$ if there exists a complete, transitive, monotone, and reflexive preference relation $\succeq$ over $Z$ and an attribute filter $\Gamma$ such that $C(S)=\{x \in \Gamma(S) \mid x \succeq y$ for every $y \in \Gamma(S)\}$.

By using a similar argument to the one preceding Definition 3, we can come up with the revealed preference associated with the model that allows for indifference.

DEFINITION 12: $x R$ if there exists a set $S$ including $y$ such that $x \in C(S)$ and for each attribute $i$ there exists a set $T^{i}$ and an alternative $t^{i}$ such that $y_{i} \geq t_{i}^{i}$, $t^{i} \in C\left(T^{i}\right)$, and

- If $z \in T^{i} \backslash S$, then $z_{i}>t_{i}^{i}$.

- If $z \in S \backslash T^{i}$, then $y_{i}>z_{i}$.

We say $x S y$ if $x>_{D} y$ or if the above condition is satisfied, but $y \notin C(S)$.

We say that $R$ satisfies only weak cycles if given any $x_{1}, x_{2}, \ldots, x_{n} \in Z$ whenever $x_{1} R x_{2} R \cdots x_{n} R x_{1}$, for no $j=1, \ldots, n-1$ can it be the case that $x_{j} S x_{j+1}$ nor can it be the case that $x_{n} S x_{1}$. As the underlying preference is transitive, only weak cycles is a necessary condition for $C$ to be CAF. The following theorem states that it is also sufficient.

THEOREM 4: A choice correspondence $C$ is $C A F$ if and only if $R$ satisfies only weak cycles.

\section{PROOF:}

Necessity already shown. For sufficiency, observe that since $R$ satisfies only weak cycles, there exists a complete preorder extension of $R$. Let $\succeq^{R}$ be one such extension; note that $\succeq^{R}$ is transitive, reflexive, complete, and monotone. The rest of the proof follows the same lines as the proof of Theorem 1.

\section{ApPendix B. ProOFs}

\section{A. Attribute Filters for Examples 1 to 5}

Example 1 (Rational Choice): For each $S, t^{S}=(0,0, \ldots, 0)$, i.e., everything is considered in any set $S$.

Example 2 ( $\operatorname{Top} n)$ : Suppose the DM considers the top $n$ alternatives in attribute 1 . Let $z^{n+1}(S)$ be the $(n+1)$ th best alternative in set $S$ according to attribute 1 if $|S| \geq n+1$, otherwise let $z^{n+1}(S)=0$. Then $t^{S}=\left(z_{1}^{n+1}(S), 0, \ldots, 0\right)$. 
Example 3 (Eliminate Worst $n$ on Each Attribute): Let $z_{i}^{l}(S)$ be the value of the $l$ th worst alternative in attribute $i$ in set $S$ if $l>0$, otherwise $z_{i}^{l}(S)=0$. Let $t^{S}(l)=\left(z_{1}^{l}(S), z_{2}^{l}(S), \ldots, z_{k}^{l}(S)\right)$. Let $l^{*}$ be the largest integer smaller or equal to $n$ such that the consideration set induced by $t^{S}\left(l^{*}\right)$ is non-empty. Then $t^{S}=t^{S}\left(l^{*}\right)$.

Example 4 (Fixed Cutoff): Without loss of generality let the ordering of the attributes be $\{k, k-1, \ldots, 1\}$. Let $t^{S}(l)$ be such that $t_{i}^{S}(l)=a_{i}^{*}$ for $i<l$ and $t_{i}^{S}(l)=0$ for $i \geq l$. Let $l^{*}$ be the largest integer smaller than or equal to $k+1$ for which the consideration set induced by $t^{S}\left(l^{*}\right)$ is non-empty. Then $t^{S}=t^{S}\left(l^{*}\right)$.

Example 5 (Satisficing Simon 1955): Assume that the DM searches through the ranking of attribute 1 . Let $N_{1}(S)=\left\{x \in S \mid \exists y \in S\right.$ such that $y_{1}>x_{1}$ and $\left.y \succ a^{*}\right\}$. Let $n_{1}(S)$ be the element of $N_{1}(S)$ with the highest value of attribute 1 if $N_{1}(S) \neq \varnothing$, otherwise let $n_{1}(S)=0$. Then $t^{S}=\left(n_{1}(S), 0,0, \ldots, 0\right)$.

\section{B. Proofs}

For the proofs, it will be useful to separate the condition in Definition 3.

CONDITION $1\left(R N_{i}\right): y \in S$ satisfies $R N_{i}$ for attribute $i$ in set $S$ if there exists a set $T$ such that either $c(T)=y$ or $y_{i}>c(T)_{i}$ and

- If $z \in T \backslash S$, then $z_{i}>c(T)_{i}$.

- If $z \in S \backslash T$, then $y_{i}>z_{i}$.

It is immediate that Definition 3 is equivalent to Definition 13 below.

DEFINITION 13: Let $x, y \in Z$ with $x \neq y$. We say $x P$ if $x>_{D}$ yor if there exists a set $S$ containing $y$ such that $c(S)=x$, and y satisfies $R N_{i}$ in set $S$ for each attribute $i$.

LEMMA 2: Take any $S \in \mathcal{Z}$ and $x \in S$.

(i) Suppose $y \in S, y_{i}>x_{i}$, and $x$ satisfies $R N_{i}$ in set $S$. Then y satisfies $R N_{i}$ in set $S$.

(ii) If $x$ satisfies $R N_{i}$ in set $S$ and if $y_{i}<x_{i}$, then $x$ satisfies $R N_{i}$ in set $\{S \cup y\}$.

(iii) If $x$ does not satisfy $R N_{i}$ in set $S$ and if $y_{i}>x_{i}$, then $x$ does not satisfy $R N_{i}$ in set $\{S \cup y\}$.

\section{PROOF:}

Suppose $x$ satisfies $R N_{i}$ in set $S$. Then by definition, there exists a set $T$ such that either $c(T)=x$ or $x_{i}>c(T)_{i}$ and

- If $z \in T \backslash S$, then $z_{i}>c(T)_{i}$.

- If $z \in S \backslash T$, then $x_{i}>z_{i}$. 
For part $(i)$, since $y_{i}>x_{i}$, by the second bullet $y \in T$. Again, by the second bullet above, if $z \in S \backslash T$ then $x_{i}>z_{i}$, implying $y_{i}>z_{i}$. But then $y$ also satisfies $R N_{i}$ in set $S$.

For part (ii) observe that as $x$ satisfies $R N_{i}$ in set $S$ and as $\{S \cup y\}$ is obtained from $S$ by adding an alternative worse than $x$ in attribute $i, x$ trivially satisfies $R N_{i}$ in set $\{S \cup y\}$.

For part (iii), assume that $x$ satisfies $R N_{i}$ in $\{S \cup y\}$. But then, since as we go from $\{S \cup y\}$ to $S$, an alternative above $x$ is removed, we have that $x$ also satisfies $R N_{i}$ in set $S$, a contradiction.

\section{PROOF OF THEOREM 1:}

Necessity already shown, here we will show sufficiency. Suppose that $P$ is acyclic.

Step 1: (Constructing the preference)-Since $P$ is acyclic, there exists a linear order extension of $P$. Let $\succ^{R}$ be a linear order extension of $P$. Then $\succ^{R}$ is a strict, transitive, complete, and monotone preference relation.

Step 2: (Forming the consideration set)—For any set $S$, we will put the threshold of attribute $i$ so that it eliminates everything that does not satisfy $R N_{i}$ in set $S$.

Let $N R N_{i}(S) \subseteq S$ be the set of alternatives that does not satisfy $R N_{i}$ in set $S$. For any set $S$, let $t_{i}^{S}=0$ if $N R N_{i}(S)=\emptyset$, otherwise let $t_{i}^{S}=\max _{\left\{z \in N R N_{i}(S)\right\}} z_{i}$.

By Lemma $2(\mathrm{i}), x_{i} \leq t_{i}^{S}$ if and only if $x$ does not satisfy $R N_{i}$ in set $S$.

Step 3: (Show that $c(S)$ maximizes $\succ^{R}$ in $\left.\Gamma(S)\right)-c(S)$ satisfies $R N_{i}$ in every attribute $i$ in set $S$, hence $c(S) \in \Gamma(S)$. Now, we need to show that $c(S) \succ^{R}$ maximizes $\Gamma(S)$.

Toward a contradiction, suppose there exists $y \in S$ such that $y \succ^{R} c(S)$ and $y>t_{i}^{S}$ for all $i \in\{1, \ldots, k\}$. But then $y$ satisfies $R N_{i}$ in set $S$ by every attribute. By the definition of $P$ we have $c(S) P$ y implying $c(S) \succ^{R} y$, a contradiction.

Step 4: (Show that $\Gamma$ is an attribute filter, i.e., elimination sets satisfy $(A),(B)$, and $(C)$ in Definition 1.)

(A) follows by construction.

To show $(B)$, assume that $y_{i} \geq t_{i}^{S}$ and $F=\{S \cup y\}$. Toward a contradiction, assume $t_{i}^{F}<t_{i}^{S}$ or $t_{i}^{F} \geq \bar{y}_{i}(S)$. In the former case, there exists $z \in F$ such that $z$ satisfies $R N_{i}$ in set $F, z$ does not satisfy $R N_{i}$ in set $S$ and $y_{i}>z_{i}$. This is a contradiction to Lemma 2(iii). In the latter case, there exists $z \in S$ that satisfies $R N_{i}$ in $S$ with $z_{i}>y_{i}$, but $z$ does not satisfy $R N_{i}$ in set $F$, which is a contradiction to Lemma $2(i i)$.

To show $(C)$, assume that $y_{i} \leq t_{i}^{S}$ and $F=\{S \cup y\}$. Toward a contradiction, assume $t_{i}^{F}>t_{i}^{S}$ or $t_{i}^{F}<y_{i}(S)$. In the former case, there exists $z \in S$ that satisfies $R N_{i}$ in $S$ with $z_{i}>y_{i}$, but $z$ does not satisfy $R N_{i}$ in set $F$, which is a contradiction to Lemma 2(ii). In the latter case, there exists $z \in F$ such that $z$ satisfies $R N_{i}$ in set $F$, $z$ does not satisfy $R N_{i}$ in set $S$ and $y_{i}>z_{i}$. This is a contradiction to Lemma 2(iii).

Step 5: (The result) - We have established that $\Gamma$ is an attribute filter and $c(S) \succ^{R}$-maximizes $\Gamma(S)$. Therefore $\left(\Gamma, \succ^{R}\right)$ rationalizes the choice. 


\section{PROOF OF THEOREM 2:}

We already showed that if $x P_{R} y$, then $x$ is revealed to be preferred to $y$. For the other direction, suppose that it is not the case that $x P_{R} y$, then there exists an extension of $P_{R}$, say $\succ_{R}$, that ranks $y$ over $x$. The proof of Theorem 1 shows that there exists an attribute filter $\Gamma_{R}$ such that $\left(\Gamma_{R}, \succ_{R}\right)$ rationalizes the choice as CAF.

We have also shown that if $x$ satisfies the condition in Definition 3 , then $x$ is revealed to be considered. For the other direction, suppose that $x$ does not satisfy the condition in set $S$, then again the proof of Theorem 1 shows that there exists a consideration set that rationalizes the choice and that does not include $x$.

LEMMA 3: If a choice function $c$ is CAFTE, then $c$ satisfies Attraction or Compromise, Attraction Consistency, and Monotonicity.

\section{PROOF:}

Suppose $c$ is CAFTE. Since $c$ is CAF, Monotonicity is trivially satisfied.

Take any $S \subseteq Z$, suppose $c(S)=x \neq c(S \cup z)=y \neq z$, without loss of generality assume $x_{2}>y_{2}$ and $y_{1}>x_{1}$. Trivially, we have $y_{2}>t_{2}^{\{S \cup z\}}$ and since $c$ is CAFTE, we have $t_{2}^{S} \geq y_{2}$. But then by $(B)$ and $(C)$ in Definition $1, z_{2}<y_{2}$. Similarly, $x_{1}>t_{1}^{S}$ and since $c$ is CAFTE, we have $x_{1} \leq t_{1}^{\{S \cup z\}}$. But then by $(B)$ and $(C)$ in Definition $1, z_{1}>x_{1}$. These two observations show that Attraction or Compromise is satisfied.

We need to show Attraction Consistency. So, suppose $y>_{D} z$ and there exists $z^{\prime} \in S$ with $z^{\prime}>_{D} z$. Assume that $y \ngtr_{D} z^{\prime}$, we will show that $y$ is in between $z^{\prime}$ and $x$. First suppose $z_{2}^{\prime}>y_{2}$, but then since $c$ is CAFTE we have $z_{1}^{\prime} \leq t_{1}^{\{S \cup z\}}$. Also, since $x_{1}>t_{1}^{S}$, by $(A), z_{1}^{\prime}>t_{1}^{S} \cdot 11 \mathrm{By}(B)$ and $(C)$ this implies that $z_{1}>z_{1}^{\prime}$, a contradiction. So, $y_{2}>z_{2}^{\prime}$. Since $y \ngtr_{D} z^{\prime}$, we also have $z_{1}^{\prime}>y_{1}$. But then, $z_{1}^{\prime}>y_{1}>x_{1}$ and $x_{2}>y_{2}>z_{2}^{\prime}$.

LEMMA 4: Suppose the choice function c satisfies Attraction or Compromise. If $c(S)=x$ and $T \subseteq S$ is obtained from $S$ by removing alternatives that rank better than $x$ in attribute $i$, then $x_{i} \geq c(T)_{i}$.

\section{PROOF:}

Toward a contradiction, suppose $c(T)_{i}>x_{i}$. As we go from $T$ to $S$, we are adding alternatives that rank better than $x$ in attribute $i$. Assume that one by one we start adding these alternatives. Let $z$ be the first alternative that leads to a choice change and $F$ be the resulting set. Then either $c(F)=z$ or the choice change is due to (i) in Attraction or Compromise or (ii) in Attraction or Compromise. But, in all of these cases, $c(F)_{i}>x_{i}$ because both $z_{i}>x_{i}$ and $c(T)_{i}>x_{i}$. But then, we can continue adding alternatives until we reach set $S$ and by the same argument the choice will never change in favor of $x$. A contradiction.

\footnotetext{
${ }^{11}$ This is because $z_{1}^{\prime}>x_{1}$. And that is because by Attraction or Compromise (which is shown to hold above) $x \ngtr_{D} z\left(\right.$ so $\left.z_{1}>x_{1}\right)$, but $z^{\prime}>_{D} z$.
} 


\section{PROOF OF THEOREM 3:}

By Lemma 3 above, if $c$ is CAFTE, then $c$ satisfies the axioms. Here, we will show the other direction. Suppose that $c$ satisfies the axioms. Let $\succ$ be any monotone preference relation and let the thresholds be defined as in the proof of Theorem 1. From the proof of Theorem 1, we already know that these lead to an attribute filter. So, all we need to show is that for any $S \subseteq Z$, the resulting consideration set $\Gamma(S)$ satisfies $\left|\Gamma(S)^{n d}\right|=1.12$

Toward a contradiction, suppose not. So, there exists $S \subseteq Z$ with $c(S)=x$, but there exists $y \in \Gamma(S), y \neq x$ that is not dominated by $x$. Since the choice satisfies Monotonicity, $x$ is also not dominated by $y$, so without loss of generality assume $x_{2}>y_{2}$ and $y_{1}>x_{1}$. Then $y$ satisfies $R N_{2}$ in set $S$. Then, by the definition of $R N_{2}$, there exists a set $T$ such that $c(T)=z, y_{2} \geq z_{2}$ and

- If $z^{\prime} \in T \backslash S$, then $z_{2}^{\prime}>z_{2}$.

- If $z^{\prime} \in S \backslash T$, then $y_{2}>z_{2}^{\prime}$.

Let $F$ be the set obtained from $T$ by removing everything that is not in $S$ and that ranks above $z$ in attribute 2 . By Lemma $4, z_{2} \geq c(F)_{2}$. Furthermore, since $c(F)$ cannot be dominated, we have $c(F)_{1} \geq y_{1}$.

Set $S$ is obtained from $F$ by adding alternatives that rank worse than $y$ in attribute 2. Divide this set into two: $(S \backslash F)_{D}$, which includes all the alternatives in $(S \backslash F)$ that are dominated by $x$ and $(S \backslash F)_{U}$, which includes the rest.

Note that everything in $(S \backslash F)_{D}$ is also dominated by $y$. Start adding the alternatives in $(S \backslash F)_{D}$ to set $F$ in any order, let $z^{1}$ be the first alternative that leads to a choice change and let $Z_{1}$ be the corresponding set. As $z^{1}$ is dominated, the choice cannot be $z^{1}$. If the choice change is due to (i) in Attraction or Compromise, then $c\left(Z_{1}\right)_{1} \geq y_{1}$. This is because of Attraction Consistency, as both $x$ and $y$ dominate $z^{1}$, but $y$ is in between $c(F)$ and $x, x$, or anything that ranks worse than $y$ in attribute 1 cannot be chosen. Finally, if the choice change is due to (ii) in Attraction or Compromise, then $c(F)_{1}>c\left(Z_{1}\right)_{1}>z_{1}^{1}$ and $z_{2}^{1}>c\left(Z_{1}\right)_{2}>c(F)_{2}$. Note that as $c\left(Z_{1}\right)$ cannot be dominated by $y$, we also have $c\left(Z_{1}\right)_{1}>y_{1}$. So, in either case $c\left(Z_{1}\right)_{1} \geq y_{1}$.

Now, we can continue adding the remaining alternatives in $(S \backslash F)_{D}$ in any order; whenever there is a choice change, the same argument shows that the chosen alternative ranks at least as good as $y$ in attribute 1 , but then $c\left(F \cup(S \backslash F)_{D}\right)_{1} \geq y_{1}$.

Note that everything in $(S \backslash F)_{U}$ ranks better than $x$ in attribute 1, so by Lemma 4, $c\left(S \backslash(S \backslash F)_{U}\right)_{1} \leq x_{1}$.

But then we have $c\left(S \backslash(S \backslash F)_{U}\right)_{1} \leq x_{1}<y_{1} \leq c\left(F \cup(S \backslash F)_{D}\right)_{1}$ $=c\left(S \backslash(S \backslash F)_{U}\right)_{1}$. The last inequality is because $\left(S \backslash(S \backslash F)_{U}\right)=\left(F \cup(S \backslash F)_{D}\right)$. A contradiction.

\footnotetext{
${ }^{12}$ Note that if this holds, then maximization of the preference trivially holds.
} 
PROOF OF LEMMA 1:

In de Clippel and Eliaz (2012), it is shown that $C^{f}(\cdot)$ satisfies the following axioms.

NO BETTER COMPROMISE: If $x, y \in C^{f}(S)$ for some set $S$, then there does not exist $z \in S$ with $y_{i}>z_{i}>x_{i}$ and $x_{j}>z_{j}>y_{j}$, where $i, j \in\{1,2\}, i \neq j$.

REMOVING AN ALTERNATIVE: If $C^{f}(S) \neq\{x\}$, then $C^{f}(S \backslash x) \cap C^{f}(S) \neq \emptyset$.

I will prove the lemma by showing that $c^{1-d f}(\cdot)$ satisfies the axioms, the result will follow by Theorem 3 . First, note that the fallback bargaining solution and hence, $c^{1-d f}(\cdot)$ trivially satisfies Monotonicity.

For the other two axioms, assume that $c^{1-d f}(S)=x, c^{1-d f}(S \cup z)=y$, where $y \neq z$ and $y \neq x$. Note that $\left|C^{f}(T)\right| \leq 2$ for all $T \in \mathcal{Z}$. Then, by Removing an Alternative, either $C^{f}(S)=\{x, y\}$ or $C^{f}(S \cup z)=\{x, y\}$.

If $C^{f}(S)=\{x, y\}$, then $x_{1}>y_{1}$ and $y_{2}>x_{2}$. Furthermore, the minimum of the scores of $x$ and $y$ are equal in $S$, whereas $y$ 's minimum score is higher in $\{S \cup z\}$, implying that $s_{1}(y, S \cup z)>s_{2}(x, S)=s_{1}(y, S)$. Inclusion of $z$ increases $s_{1}(y, S)$, so $x_{1}>y_{1}>z_{1}$; furthermore, it does not increase $s_{2}(x, S)$, so $z_{2}>x_{2}$. But then either $y$ asymmetrically dominates $z$ or $y$ is in between $x$ and $z$, hence Attraction or Compromise is satisfied.

If $C^{f}(S \cup z)=\{x, y\}$, then $y_{1}>x_{1}$ and $x_{2}>y_{2}$. Furthermore, the minimum of the scores of $x$ and $y$ are equal in $\{S \cup z\}$, whereas $y$ 's minimum score is lower in $S$, implying that $s_{2}(y, S)<s_{1}(x, S)=s_{1}(x, S \cup z)$. Removal of $z$ reduces $s_{2}(y, S)$, so $x_{2}>y_{2}>z_{2}$; furthermore, it does not reduce $s_{1}(x, S)$, so $z_{1}>x_{1}$. But then either $y$ asymmetrically dominates $z$ or $y$ is in between $x$ and $z$, hence Attraction or Compromise is satisfied in both of the cases.

To see that Attraction Consistency is satisfied, assume that $y>_{D} z$, but there exists another alternative $z^{\prime} \in S$ with $z^{\prime}>_{D} z$. By No Better Compromise, $z^{\prime}$ cannot be in between $x$ and $y$, and by Monotonicity, it can neither dominate $x$ nor $y$. But then, either $y$ is in between $z^{\prime}$ and $x$ or $y$ dominates $z^{\prime}$. Hence, Attraction Consistency is satisfied.

Since $c^{1-d f}(\cdot)$ satisfies Attraction or Compromise, Attraction Consistency, and Monotonicity, it is CAFTE.

\section{REFERENCES}

Brams, Steven J., and D. Marc Kilgour. 2001. "Fallback Bargaining." Group Decision and Negotiation 10 (4): 287-316.

Caplin, Andrew, and Mark Dean. 2011. "Search, choice, and revealed preference." Theoretical Economics 6 (1): 19-48.

Caplin, Andrew, Mark Dean, and Daniel Martin. 2011. "Search and Satisficing." American Economic Review 101 (7): 2899-2922.

Cherepanov, Vadim, Timothy Feddersen, and Alvaro Sandroni. 2013. "Rationalization." Theoretical Economics 8 (3): 775-800.

de Clippel, Geoffroy, and Kfir Eliaz. 2012. "Reason-based choice: A bargaining rationale for the attraction and compromise effects." Theoretical Economics 7 (1): 125-62.

Eliaz, Kfir, and Ran Spiegler. 2011a. "Consideration Sets and Competitive Marketing." Review of Economic Studies 78 (1): 235-62. 
Eliaz, Kfir, and Ran Spiegler. 2011b. "On the strategic use of attention grabbers." Theoretical Economics 6 (1): 127-55.

Gabaix, Xavier, David Laibson, Guillermo Moloche, and Stephen Weinberg. 2006. "Costly Information Acquisition: Experimental Analysis of a Boundedly Rational Model." American Economic Review 96 (4): 1043-68.

Gerasimou, Georgios. 2016. "Asymmetric dominance, deferral, and status quo bias in a behavioral model of choice." Theory and Decision 80 (2): 295-312.

Hauser, John R., and Birger Wernerfelt. 1990. "An Evaluation Cost Model of Consideration Sets." Journal of Consumer Research 16 (4): 393-408.

Huber, Joel, John W. Payne, and Christopher Puto. 1982. "Adding Asymmetrically Dominated Alternatives: Violations of Regularity and the Similarity Hypothesis." Journal of Consumer Research 9 (1): 90-98.

Kıbrıs, Özgür, and Murat R. Sertel. 2007. "Bargaining over a finite set of alternatives.” Social Choice and Welfare 28 (3): 421-37.

Lleras, Juan Sebastián, Yusufcan Masatlioglu, Daisuke Nakajima, and Erkut Y. Ozbay. 2017. "When more is less: Limited consideration." Journal of Economic Theory 170: 70-85.

Lombardi, Michele. 2009. "Reason-based choice correspondences." Mathematical Social Sciences 57 (1): 58-66.

Mandler, Michael, Paola Manzini, and Marco Mariotti. 2012. "A million answers to twenty questions: Choosing by checklist." Journal of Economic Theory 147 (1): 71-92.

Manzini, Paola, and Marco Mariotti. 2007. "Sequentially Rationalizable Choice." American Economic Review 97 (5): 1824-39.

Manzini, Paola, and Marco Mariotti. 2012. "Categorize Then Choose: Boundedly Rational Choice and Welfare." Journal of the European Economic Association 10 (5): 1141-65.

Manzini, Paola, and Marco Mariotti. 2014. "Stochastic Choice and Consideration Sets." Econometrica 82 (3): 1153-76.

Masatlioglu, Yusufcan, and Daisuke Nakajima. 2013. "Choice by iterative search." Theoretical Economics 8 (3): 701-28.

Masatlioglu, Yusufcan, Daisuke Nakajima, and Erkut Y. Ozbay. 2012. "Revealed Attention." American Economic Review 102 (5): 2183-2205.

Natenzon, Paulo. Forthcoming. "Random Choice and Learning." Journal of Political Economy.

Ok, Efe A., Pietro Ortoleva, and Gil Riella. 2015. "Revealed (P)Reference Theory." American Economic Review 105 (1): 299-321.

Roberts, John H., and James M. Lattin. 1991. "Development and Testing of a Model of Consideration Set Composition." Journal of Marketing Research 28 (4): 429-40.

Simon, Herbert A. 1955. “A Behavioral Model of Rational Choice." Quarterly Journal of Economics 69 (1): 99-118.

Simonson, Itamar. 1989. "Choice Based on Reasons: The Case of Attraction and Compromise Effects." Journal of Consumer Research 16 (2): 158-74.

Sprumont, Yves. 1993. "Intermediate preferences and Rawlsian arbitration rules." Social Choice and Welfare 10 (1): 1-15.

Tversky, Amos. 1972. "Elimination by aspects: A theory of choice." Psychological Review 79 (4): 281-99.

Tversky, Amos, and Itamar Simonson. 1993. "Context-dependent Preferences.” Management Science 39 (10): 1179-89.

Wright, Peter, and Fredrick Barbour. 1977. "Phased Decision Strategies: Sequels to an Initial Screening." Stanford Graduate School of Business Working Paper 353. 
Copyright of American Economic Journal: Microeconomics is the property of American Economic Association and its content may not be copied or emailed to multiple sites or posted to a listserv without the copyright holder's express written permission. However, users may print, download, or email articles for individual use. 